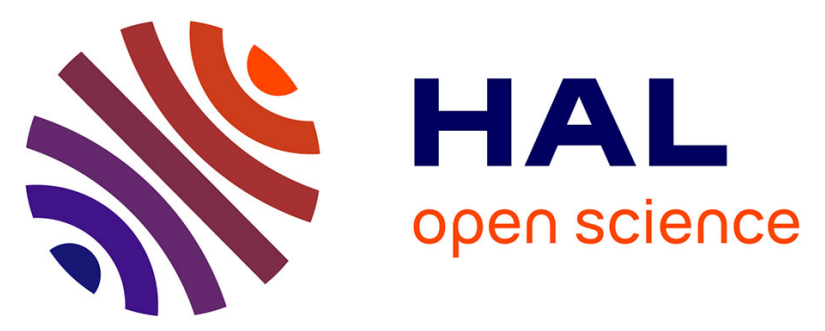

\title{
Printing functional protein nano-dots on soft elastomers: from transfer mechanism to cell mechanosensing
}

Ranime Alameddine, Astrid Wahl, Fuwei Pi, Kaoutar Bouzalmate, Laurent Limozin, Anne Charrier, Kheya Sengupta

\section{- To cite this version:}

Ranime Alameddine, Astrid Wahl, Fuwei Pi, Kaoutar Bouzalmate, Laurent Limozin, et al.. Printing functional protein nano-dots on soft elastomers: from transfer mechanism to cell mechanosensing. Nano Letters, 2017, 17 (7), pp.4284 - 4290. 10.1021/acs.nanolett.7b01254 . hal-01774028

\section{HAL Id: hal-01774028 \\ https://hal.science/hal-01774028}

Submitted on 23 Apr 2018

HAL is a multi-disciplinary open access archive for the deposit and dissemination of scientific research documents, whether they are published or not. The documents may come from teaching and research institutions in France or abroad, or from public or private research centers.
L'archive ouverte pluridisciplinaire HAL, est destinée au dépôt et à la diffusion de documents scientifiques de niveau recherche, publiés ou non, émanant des établissements d'enseignement et de recherche français ou étrangers, des laboratoires publics ou privés. 


\section{Printing functional protein nano-dots on soft elastomers: from transfer mechanism to cell mechanosensing \\ Ranime Alameddine, ${ }^{\dagger}$ Astrid Wahl, ${ }^{\dagger}$ Fuwei $\mathrm{Pi}^{\dagger},{ }^{\dagger}$ Kaoutar Bouzalmate, ${ }^{\dagger}$ Laurent Limozin,${ }^{\ddagger}$ Anne \\ Charrier, ${ }^{\dagger}$ and Kheya Sengupta ${ }^{*} \dagger$ \\ $\dagger$ Aix Marseille University, CNRS, CINaM-UMR 7325, Marseille, 13288, France \\ $\ddagger$ Laboratoire Adhesion Inflammation, Aix-Marseille University, UMR INSERM 1067, UMR CNRS 7333, \\ 13288 Marseille, France \\ \Current address:State Key Laboratory of Food Science and Technology,School of Food Science, Jiangnan University, Wuxi, Jiangsu 214122, China. \\ Received March 24, 2017; E-mail: sengupta@cinam.univ-mrs.fr.}

\begin{abstract}
Living cells sense the physical and chemical nature of their micro/nano environment with exquisite sensitivity. In this context, there is a growing need to functionalize soft materials with micro/nano-scale bio-chemical patterns for applications in mechanobiology. This however is still an engineering challenge. Here a new method is proposed, where sub-micronic proteinpatterns are first formed on glass and are then printed on to an elastomer. The degree of transfer is shown to be governed mainly by hydrophobic interactions and to be influenced by grafting an appropriate fluorophore onto the core protein of interest. The transfer mechanism is probed by measuring the forces of adhesion/cohesion using atomic force microscopy. The transfer of functional arrays of dots with size down to about 400 $\mathrm{nm}$, on elastomers with stiffness ranging from $3 \mathrm{kPa}$ to $7 \mathrm{MPa}$, is demonstrated. Pilot studies on adhesion of $\mathrm{T}$ lymphocytes on such soft patterned substrates are reported.
\end{abstract}

In the last decades more and more experiments have confirmed that living cells are sensitive to the mechanics of their immediate environment. They behave differently on stiff and soft substrates in terms of adhesion, migration, actin organization, force generation, differentiation and a host of other properties. ${ }^{1-5}$ In separate studies, it was shown that adherent cells respond to the way adhesive ligands are distributed or grouped into micro or nano-patterns. ${ }^{6-9}$ Single molecules of adhesive ligands that are separated by distances larger than a cut-off length scale of about $80 \mathrm{~nm}$ fail to support adhesion, spreading, proliferation and growth of connective tissue cells like fibroblasts. ${ }^{7,10}$ Intriguingly, the behavior of lymphocyte-like cells may depend not on ligand spacing but on average ligand density. ${ }^{9,11-13}$ Directing cell behavior and fate, in a cell type and ligand dependent manner and through physical means, is of major interest for tissue-engineering applications. ${ }^{14}$

In the general context of cell biology as well as the specific context of lymphocytes, there is therefore a lot of interest in patterning of soft substrates but even at the micro-scale this is a current engineering challenge. ${ }^{15,16}$ At the nanoscale there is, so far, only one available technique that has been applied to cell studies, ${ }^{17}$ which is based on patterning soft hydrogels employing block copolymer micelle lithography (BCML) for the production of surfaces patterned with gold nanoparticles on a hard template, then transfer the pattern to a polymeric hydrogel. ${ }^{18,19}$ This technique is therefore dependent on the chemistry and optics of gold and is limited to the visco-elastic range of the hydrogel. Another technique with the potential to achieve nano-scale features on a hydrogel is deep UV etching followed by transfer, but so far this has been mainly confined to larger, micron size patterns and is also limited by the visco-elastic range of the hydrogel. ${ }^{20,21}$ Moreover, in the view of seminal work dissecting possible differences between cells on hydrogels and on elastomers, ${ }^{14}$ patterning both types of material is important.

A large number of techniques have been devised to nanopattern hard inorganic substrates like glass or silicon, ${ }^{22,23}$ including photo or electron-beam lithography, ${ }^{24}$ dip pen nanolithography, ${ }^{25,26}$ and nano-imprint technologies. ${ }^{10,27}$ An universal technique to transfer such patterns to soft substrates will address a specific need in the community. Micro and nano contact printing, which uses elastomeric stamps with a pattern of relief structures to transfer molecules to glass or gold surface, is now an ubiquitous tool in biology. ${ }^{6,28-35}$ These techniques, and their offshoots, depend on the high affinity of the ink molecules for the target surface which is typically glass or gold. The reverse transfer of protein patterns from glass to elastomer was demonstrated at micron-scale on Polydimethylsiloxane (PDMS) but was limited by a requirement of treatment of PDMS leading to its hardening. ${ }^{36,37}$ Similar transfer to soft native PDMS turned out to be possible, ${ }^{38,39}$ but only under very special conditions. Here we present the key idea that by tuning molecular affinities of the transferred proteins via grafting of selected chemical moieties, the reverse transfer from glass to an elastomer can be systematically controlled. We demonstrate the transfer at sub-micron scale onto very soft PDMS and present pilot experiments with cells. We show that the transfer can be predicted from measured adhesive/cohesive forces between the protein molecules and the glass/PDMS surface. We argue that the degree of hydrophobicity is the major, and the presence of ionic groups is a minor factor that governs the transfer.

The pattern and transfer process is presented schematically in Figure 1. In brief, to pattern glass, cover-slides were thoroughly washed and a colloidal bead mask was formed on them as described before. ${ }^{38}$ A fluorosilane (Trichloro(1H,1H,2H,2H-perfluorooctyl)silane, PFOTCS) was deposited from the vapor phase on the glass through the bead-mask. The beads were removed, revealing a layer of hydrophobic fluorosilane patterned with holes. The hydrophobic regions were passivated by absorption of a triblock poloxamer (chosen to be Pluronic F68, see SI). The holes exposing bare glass were subsequently back-filled with a protein of choice. The protein of choice here is variously functionalized bovine serum albumin (BSA) which was usu- 
ally biotinylated (bBSA) and additionally labeled with a fluorophore, or neutravidin (NAV) also conjugated to a fluorophore. As examples, we chose two well known fluorophores : the hydrophobic dye Texas Red (TR) and the hydrophillic dye Atto-488 (Atto). The binding steps are schematically presented in Figure 1, and were checked by imaging with atomic force microscopy (AFM) (Figure SI.1). At this stage, the cover-slide is chemically patterned with protein nano-dots, and is henceforth called the glass-master.

\begin{tabular}{ll|l}
\hline \multicolumn{2}{c|}{ Fabrication of the glass master } & Transfer of the protein pattern to PDMS \\
\hline (a) & Silane $(\leq 1 \mathrm{~nm})$ \\
\hline (b) & (c) \\
\hline
\end{tabular}

Figure 1. Schematic representation of the fabrication of protein nano-patterns on glass and soft substrates: (a) Deposition of fluorosilane from a gas phase through a self-assembled colloidal bead mask on a glass substrate. (b) Removal of the mask and grafting of a poloxamer (pluronic) to passivate the fluorosilane covered area. (c) Functionalization of the bare patches with the desired protein. (d) Thin layer of elastomer supported on a glass coverslide. (e) Transfer of the protein from glass to elastomer by reverse contact printing in presence of water. (f) Protein pattern on the elastomer.

A second glass cover-slide was coated with a layer of elastomer precursor solution by spin-coating and was appropriately cured. The elastomers used here include: a polydimethyl based silicone rubber (corresponding to sylgard184 used with base to cross-linker ratio of 10:1, henceforth called PD), or a silicone gel containing additional methylphenyl groups and phenyl polymers (corresponding to Q-gel 920 used with ratio of $1: 2$, henceforth called MP), or, for certain experiments, another polydimethyl based silicone rubber (corresponding to CY 52-276, henceforth called PD2). All three types of elastomers were either used as is after curing or were exposed to oxygen plasma (accordingly, the latter are henceforth called pPD, pMP or pPD2). SI table 1 and 2 report the Young modulus and contact angle measurements on the different surfaces, showing that a stiffness range of about $3 \mathrm{kPa}$ to $7 \mathrm{MPa}$ was covered and that the elastomers can be arranged in order of hydrophobicity as $\mathrm{PD} 2 \approx \mathrm{MP}>\mathrm{PD} \gg \mathrm{pMP}>\mathrm{pPD} 2>\mathrm{pPD}$. For some experiments, the plasma exposed PDMS was further functionalized with a organo-aminosilane and glutaraldehyde (gluMP and gluPD2), re-rendering them hydrophobic.

The surface of the elastomer layer was brought into physical contact with the glass-master in presence of a drop of water. The presence of water, in agreement with other reports ${ }^{40}$ facilitates the transfer. A minimal pressure was applied manually to ensure conformal contact. Since both the surfaces are flat and do not appreciably deform on contact, pressure is not a crucial parameter and it was verified that the quality of transfer is not sensitive to the applied pressure. The surfaces were carefully separated the next day to obtain the patterned elastomer. The patterned surface was imaged using epi-fluorescence microscopy (Figure 2) and AFM (Figure SI.2).

Visual inspection of images in Figure 2 reveals that bBSA functionalized with Texas Red dye (bBSA-TR) transferred on $\operatorname{PD}(a, b)$ but the same core protein functionalized with Atto-488 dye (hbBSA-Atto) failed to transfer (c,d). bBSAAtto could however be transferred to pPD (e,f). We note that on one hand, from the molecular structure of Atto and TR we expect TR to be more hydrophobic than Atto, the latter having an isolated primary amine group that is prone to losing an anion. On the other hand, native PD is hydrophobic but plasma treated pPD is hydrophillic. Thus, bBSA could be transferred to native hydrophobic PDMS if conjugated to a hydrophobic dye, and to hydrophillic PDMS if conjugated to a hydrophillic dye. These observations lead us to test a variety of proteins and elastomer surfaces. The table in Figure 2 summarizes the success, or not, of the attempted transfers. As conjectured, proteins (bBSA, BSA or NAV) labeled with hydrophobic dyes transfer well on hydrophobic PDMS: either native or glutaraldehyde treated (PD,MP,PD2, gluMP,gluPD2), whereas bBSA labeled with hydrophilic dyes transfers well on hydrophilic, plasma treated elastomers (pPD,pMP). Cross transfer is possible only in some cases.
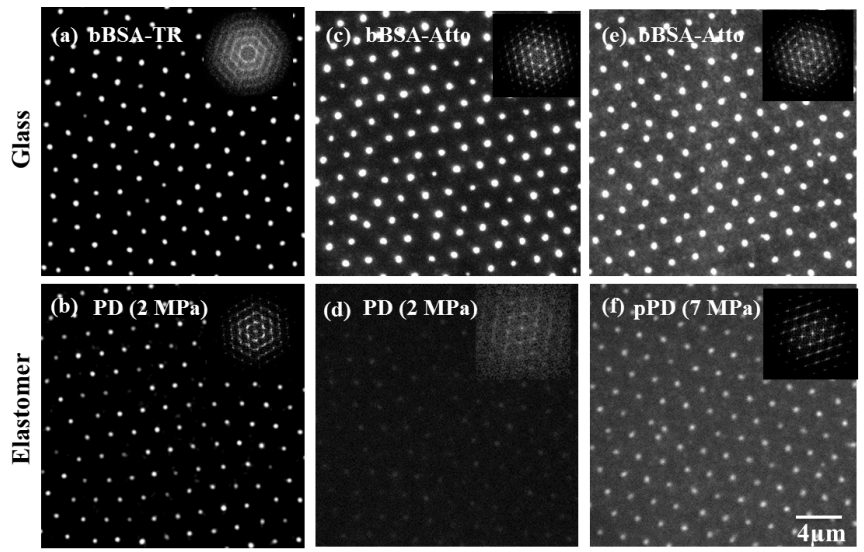

\begin{tabular}{|c|c|c|c|c|c|c|c|}
\hline Proteins & $\begin{array}{r}\mathrm{MP} \\
20 \mathrm{kPa}\end{array}$ & $\begin{array}{l}\mathrm{PD} \\
2 \mathrm{MPa}\end{array}$ & $\begin{array}{l}\mathrm{PD} 2 \\
3 \mathrm{kPa}\end{array}$ & glu MP & Glu PD2 & $\begin{array}{c}\mathrm{pMP} \\
120 \mathrm{kPa}\end{array}$ & $\begin{array}{l}\mathrm{pPD} \\
7 \mathrm{MPa}\end{array}$ \\
\hline bBSA-Atto & v & $x$ & & & & (v) & (v) \\
\hline bBSA & $v$ & & $x$ & $x$ & $\mathrm{x}$ & & \\
\hline BSA-FITC & $x$ & $\mathbf{x}$ & $\mathrm{x}$ & $x$ & $x$ & (v) & \\
\hline bBSA-TR & (v) & (v) & & & & $v$ & $v$ \\
\hline BSA-TR & (v) & (v) & & & & & \\
\hline Nav-TR & (v) & (v) & (v) & (v) & ( & & \\
\hline
\end{tabular}

Figure 2. TOP: Epi-fluorescence images of protein dots on glass master before transfer and on elastomer after transfer with same camera and display settings for all images. (a,b) bBSA-TR to PD. (c,d) bBSA-Atto to PD. (e,f) bBSA-Atto to pPD. Insets display Fourier transforms of the corresponding images to emphasize the ordering of the lattice. BOTTOM: Table summarizing the transfer of proteins to elastomer surfaces. Rows correspond to one kind of protein, the core being either BSA or NAV, which are then decorated with various fluorophores as indicated. Hydrophilic molecules are depicted in blue and hydrophobic in red. Columns correspond to different elastomers, either native (hydrophobic, in red) or plasma treated (hudrophilic, in blue). Successful transfers are indicated with $\checkmark$ and unsuccessful ones with $\times$. Note that the intersection between identically colored lines and columns invariably results in a successful transfer (red or blue circled checkmark). The intersection between differently colored lines and columns results in failed transfer in most cases $(\times$ symbol $)$.

To put these observations on a quantitative basis, we selected the transfer of bBSA-TR or bBSA-Atto onto PD,MP,pPD and pMP. Fig.SI.3 shows representative im- 
(a)
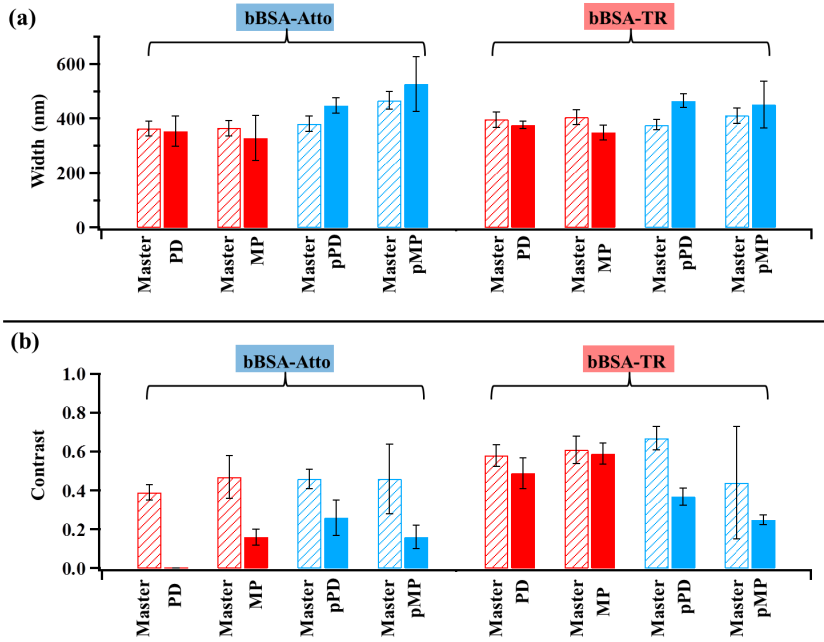

Figure 3. Quantification of the bBSA nano-dots from the epifluorescence images before and after the transfer from glass to elastomer. Values are medians and error bars are median absolute deviation, both averaged over at least 3 independent samples, each with at least 6 fields each containing hundreds of dots. (a) Dot-size (FWHM of the intensity profile). (b) Contrast of the dots with respect to the background. Data are for bBSA-Atto or bBSA-TR dots and PDMS type PD, MP,pPD or pMP, as indicated

(a)
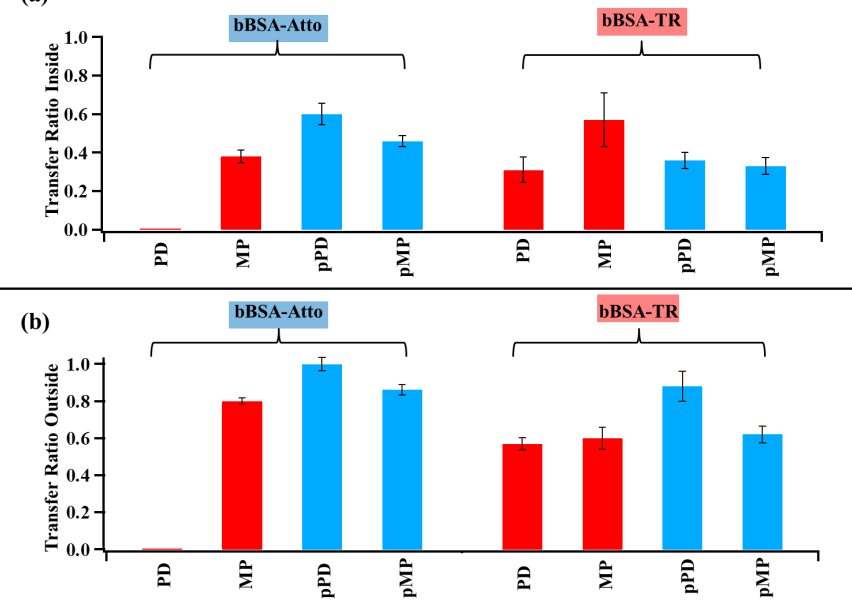

Figure 4. Transfer ratio of proteins inside the dots (a) and outside the dots (poloxamer covered zones) (b). Calculated from data presented in figure SI.5. Data are for bBSA-Atto or bBSATR dots and PDMS type PD, MP,pPD or pMP, as indicated

ages for these transfers and Fig. 2 summarizes the dot size and contrast (see SI text for details of data analysis). The parameters reported here are averages calculated from at least 3 samples, with at least 6 fields each. The transfer of the pattern to both PD and MP conserves the size, but transfer to $\mathrm{pPD}$ or pMP increases the dot size slightly ( $\mathrm{T}$ test: $\mathrm{p}<0.001)$. The contrast is systematically diminished on transfer, implying that the amount of protein transferred from within the dots is not identical to the transfer outside the dots. To characterize this, a transfer ratio was defined as $I_{\text {max }}^{\text {elastomer }} / I_{\text {max }}^{\text {glass }}$. A similar transfer ratio can be separately calculated for intensity outside the $\operatorname{dots}\left(I_{\text {min }}^{\text {elastomer }} / I_{\text {min }}^{\text {glass }}\right)$ to quantify of the amount of transfer in the polaxamer coated zones. Here, $I_{\max }$ and $I_{\min }$ are the maximum and minimum intensity in the pattern which essentially correspond to the peak intensity in the dots and the background intensity out- side the dots (see Figure ). The transfer ratio inside (Figure 4a) clearly shows that the chemical nature of the grafted fluorophore as well as the elastomer surface influences the success of transfer. For the same fluorophore and elastomer, the transfer ratio outside (Figure $4 \mathrm{~b}$ ) roughly similar to the transfer inside, and is often non-negligible. It is clear that to obtain good patterns, the transfer of proteins inside the dots should be maximal. Outside, in the ideal case, while making the glass-master, there should be no protein absorbed on the regions passivated with the poloxamer and even if there is some protein absorbed, it should not be transferred to PDMS. In practice, the amount of protein absorbed and transferred both depend on the quality of the poloxamer layer. In light of the non-negligible transfer in all cases, the strategy here was to minimize protein absorption outside the dots at the glass-master stage. The poloxamer type and concentration used was optimized accordingly (data SI Figure SI.6). In this work, an inside transfer ratio $>0.2$ is considered a successful stamping, irrespective of the outside transfer ratio.

To verify the hypothesis that the forces of adhesion, possibly originating from hydrophobic/hydrophilic affinities, govern the success of transfer, we quantified the effective force of adhesion of the protein on glass and elastomers using AFM force curves. ${ }^{41,42}$ The protein of interest was covalently bound to a glass bead attached to the AFM cantilever (Figure SI.7). The protein covered bead was approached and made to touch a test surface which was either bare clean glass, or bare elastomer, or glass covalently functionalized with the same protein. The retraction curves were analyzed in order to extract the force of adhesion. As control measurements, we also obtained force curves from intermediate steps of functionalization to ensure that the functionalization steps were correctly realized. PD turned out to have a very strong non-specific adhesion with the bead, probably due to van der Wall's interactions and these measurements were not amenable to interpretation. However, forcecurves could be consistently measured and interpreted for pPD (Figure SI.8). For MP, such measurements were difficult even after plasma treatment, probably due to its extreme softness. The force of adhesion for pPD (called $\left.F_{p P D}\right)$, glass (called $F_{\text {glass }}$ ), and protein coated glass (called $F_{\text {prt }}$ ) are summarized in table 1.

Table 1 shows that the force required to separate a protein layer from glass is less than that required to pull apart two layers of protein $\left(F_{\text {glass }}<F_{\text {prt }}\right)$. Therefore the protein multi-layers expected to be present on the dots on the glass-master are transferred to the elastomer by peeling from glass, in practice, they in fact fracture due to presence of defects. Comparing the adhesion of bBSA-TR and bBSA-Atto, we see that the latter has a stronger interaction with pPD. This is consistent with the higher transfer ratio (about 0.6 for bBSA-Atto and about 0.4 for bBSA-TR) reported for bBSA-Atto in Figure 4a.

Let us now re-examine the transfer table (Figure 1). As discussed above, the hydrophobic bBSA-TR transfers well on both $\mathrm{PD}$ and MP, with the transfer being better on the latter (TrRatio about 0.3 and 0.6 respectively). Consistent with this, hydrophilic FITC transfered to pMP. Crosstransfers (hydrophobic on hydrophilic or vice versa), may however either fail as expected or be possible due to additional considerations. As expected, the hydrophillic bBSAAtto fails to transfer to hydrophobic PD. In fact though the transfer is feeble and cannot be detected with the stan- 
dard camera settings, it can be detected with higher camera amplification (Figure SI.9). Furthermore, PD is known to be slightly negatively charged in aqueous solution at neutral $\mathrm{pH},{ }^{43}$ resulting in a additional electrostatic repulsion towards the negatively charged bBSA-Atto. Consistent with this, hydrophilic FITC conjugated bBSA failed to transfer to untreated PD2. However, bBSA-Atto does transfer to some extent (TrRatio about 0.4) to MP, which is even more hydrophobic as judged by contact angle measurements. We rationalize this observation by noting that bBSA-Atto has many phenyl groups that may chemically interact with the phenyl groups on MP through $\pi-\pi$ interactions.

The transfer of bBSA (not conjugated to any fluorophore) can not be directly tested since in absence of an attached fluorophore, bBSA can not be imaged in fluorescence microscopy. It needs to be revealed by functionalization with fluorescent neutravidin (NAV) after transfer and therefore transfer ratios can not be reported. To do this, after transfer of the bBSA, the bare elastomer around the dots was passivated with a polaxamer and then the NAV was allowed to bind from solution phase (see Figure SI.10 for example). bBSA by itself fails to transfer to PD2 and has very unreliable transfer to MP (data not shown). We conclude that on the glass master, bBSA mainly exposes hydrophilic groups, thus preventing its transfer to the hydrophobic PDMS surface.

To confirm the general hypothesis that the inclusion of a hydrophobic moiety renders a protein more amenable to transfer on hydrophobic untreated elastomers, we checked that neutravidin conjugated to Texas red dye (NAV-TR) transferred well on all the elastomers studied here. However, probably due to drying, the transferred neutravidin was not functional and failed to bind to a biotinylated protein.

In a related set of experiments we functionalized the plasma treated elastomer surface (pPD2 and pMP) with APTES ((3-Aminopropyl)triethoxysilane) and glutaraldehyde, which is known to render the surface hydrophobic. ${ }^{44}$ The hydrophobic NAV-TR, transfered well on this type of surface, but hydrophilic bBSA and BSA-FITC showed a very low transfer, again showing that the transfer is governed by physico-chemical affinity.

We also compared the impact of hardness on transfer. To minimize artifacts arising from elastomer surface chemistry, hard and soft versions of the same elastomer were employed (Figure SI.11). It is seen that the width does not significantly change in either case and that the transfer is successful in both cases as judged from the calculated transfer ratios.

We next confirmed that the bBSA-TR transferred to an elastomer could be functionalized with NAV. First, the area around the dots, still exposing the PDMS substrate, was passivated using poloxamers. Using NAV labeled with another fluorophore (dylight-650), the specific binding of NAV to bBSA-TR dots can be confirmed (Figure SI.10). The NAV can be further functionalized with another biotinylated protein, here an antibody against the CD3 domain in the TCR-complex in T lymphocytes ( $\alpha$-CD3, multibiotinylated UCHT1).

A soft elastomer, chosen to be MP with Young's modulus $20 \mathrm{kPa}$, was patterned with $\alpha$-CD3 dots and passivated with a poloxamer. $\mathrm{T}$ cells were allowed to interact with this substrate for 30 minutes and were then fixed and labeled with an antibody against TCR or with fluorescent phalloidin to label actin (SI for details). Cells are seen to adhere, as judged from reflection interference contrast microscopy (Figure 5 a), with an irregular contour but with a rather homogeneous adhesion within the contact zone. The actin is in the form of a ring (Figure $5 \mathrm{~b}$ ), as has been reported in the case of homogeneous $\alpha$-CD3 absorbed to glass. ${ }^{45}$ TCR clusters are detectable and partially co-localize with the underlying anti-CD3 dots (Figure $5 \mathrm{c}$ and $\mathrm{d}$ ). The overall behavior on patterned glass is fairly similar (Figure SI.12) . The invariance in actin and TCR organization was unexpected since for other cell types, as reported for adhesion to homogeneously distributed ligands, the cell adhesion is diminished on soft substrates and the cytoskeletal organization is strongly impacted. ${ }^{4,5}$ A comparison with homogeneously functionalized elastomer reveals that detectable micro-clusters do not form in this case. This is consistent with the difference observed between patterned and homogeneously functionalized glass substrates. $^{9}$

The absorption of proteins to synthetic surfaces is important for a number of applications. For example, a newly implanted prosthesis is first coated by proteins from the body fluids before cells can interact with it. In a laboratory setting, many biology experiments depend on successful protein absorption as the first step towards functionalization. Yet, the non-specific absorption of proteins to a surface from a solution phase is difficult to model, partly because of the complex and diverse molecular structure of the proteins. In the context of micro-contact printing, several attempts have been made to quantitatively model the transfer and yet they serve at best as indicative and most laboratories still rely on trial and error to test if their protein of interest can be stamped. In fact, it turns out that most proteins of interest readily transfer from traditional PDMS elastomer stamps to glass. Here we have shown that the reverse transfer of proteins from glass to PDMS is also possible, but depends crucially on the physico-chemical interactions between the protein and the PDMS surface.

One point of great practical significance is that proteins that can not be reverse transferred in native state, can be made to do so after by grafting an appropriate molecular moiety, here chosen to be different fluorophores. We have shown that proteins grafted with a hydrophobic moiety always transfer well on PDMS with hydrophobic surface which is the case for most available PDMS in their native state. The reverse transfer of proteins that are intrinsically hydrophillic or those that are grafted with a hydrophillic group, onto hydrophobic PDMS is not reliable. However, these typically transfer well on PDMS rendered hydrophilic by plasma treatment. Interestingly, atomic force microscopy based force measurements confirmed that indeed the degree of reverse transfer depends on the force required to detach a bead, grafted with a layer of protein, from the surface of the PDMS.

We showed that the protein pattern created by reverse printing from flat stamps remains functional and can be further functionalized with a more complex protein of choice. We demonstrated the adhesion of $\mathrm{T}$ cells to RCP-patterns functionalized with an antibody against the TCR complex. Consistent with previous observations on glass, the TCR can gather to form micro-clusters on patterned but not on homogeneously coated PDMS. The knowledge of mechanisms governing reverse transfer elucidated here should open the way to systematically using such glass based flat-stamps to pattern elastomers with any desired protein molecules.

Acknowledgement The authors thank Pierre Dillard for 

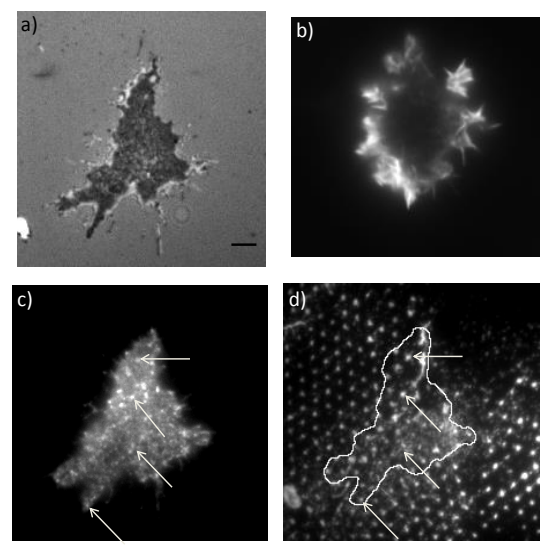

Figure 5. T-cells adhered on soft elastomer (MP) patterned with nano-dots of $\alpha$-CD3 antibody, observed after 30 minutes of spreading. a) RICM image showing a flat membrane topography in the cell adhesion zone. b) TIRF image showing peripheral actin organization. c) TIRF image of labelled TCR on the cell surface d) epi-fluorescence image of the underlying $\alpha$-CD3 dots. Arrows on $\mathrm{c}$ and d point to partial co-localization of the TCR with the $\alpha$-CD3 dots. Scale bar: $4 \mu \mathrm{m}$.

Table 1. Adhesion force measurement. Each value is extracted from 100 independent force curves taken at 3 different regions for at least 2 different samples.

\begin{tabular}{|l|c|c|c|}
\hline & $F_{\text {prt }}(\mathrm{nN})$ & $F_{\text {glass }}(\mathrm{nN})$ & $F_{p P D M S}(\mathrm{nN})$ \\
\hline BSAb TR & $4.8 \pm 1.5$ & $2.7 \pm 0.8$ & $5.1 \pm 0.6$ \\
\hline BSAb Atto 488 & $10.5 \pm 3.5$ & $2.3 \pm 0.8$ & $12.8 \pm 1.7$ \\
\hline
\end{tabular}

fruitful discussions regarding cell experiments, and Martine Biarnes for help with cell culture. This work was partially funded by European Research Council via grant no. 307104 FP/2007-2013/ERC-Stg SYNINTER.

Supporting Information Available: The file SI.pdf is available free of charge and contains tables (Young's modulus and contact angles), figures (AFM images, force-curves, untreated optical images, complementary data) and details on materials and methods. This material is available free of charge via the Internet at http://pubs.acs.org/.

\section{References}

(1) Pelham, R. J.; Wang, Y.-1. Proceedings of the National Academy of Sciences 1997, 94, 13661-13665.

(2) Flanagan, L. A.; Ju, Y.-E.; Marg, B.; Osterfield, M.; Janmey, P. A. Neuroreport 2002, 13, 2411.

(3) Discher, D. E.; Janmey, P.; Wang, Y.-1. Science 2005, 310, 1139-1143.

(4) Solon, J.; Levental, I.; Sengupta, K.; Georges, P. C.; Janmey, P. A. Biophysical journal 2007, 93, 4453-4461.

(5) Engler, A. J.; Sen, S.; Sweeney, H. L.; Discher, D. E. Cell 2006 $126,677-689$.

(6) Chen, C. S.; Mrksich, M.; Huang, S.; Whitesides, G. M.; Ingber, D. E. Science 1997, 276, 1425-1428.

(7) Cavalcanti-Adam, E. A.; Volberg, T.; Micoulet, A.; Kessler, H.; Geiger, B.; Spatz, J. P. Biophysical journal 2007, 92, 29642974.

(8) Tseng, Q.; Duchemin-Pelletier, E.; Deshiere, A.; Balland, M.; Guillou, H.; Filhol, O.; Théry, M. Proceedings of the National Academy of Sciences 2012, 109, 1506-1511.

(9) Dillard, P.; Pi, F.; Lellouch, A. C.; Limozin, L.; Sengupta, K. Integrative Biology 2016, 8, 287-301.

(10) Schvartzman, M.; Palma, M.; Sable, J.; Abramson, J.; Hu, X.; Sheetz, M. P.; Wind, S. J. Nano letters 2011, 11, 1306-1312.

(11) Deeg, J.; Axmann, M.; Matic, J.; Liapis, A.; Depoil, D. Afrose, J.; Curado, S.; Dustin, M. L.; Spatz, J. P. Nano letters 2013, 13, 5619-5626.

(12) Matic, J.; Deeg, J.; Scheffold, A.; Goldstein, I.; Spatz, J. P. Nano letters 2013, 13, 5090-5097.
(13) Delcassian, D.; Depoil, D.; Rudnicka, D.; Liu, M.; Davis, D. M.; Dustin, M. L.; Dunlop, I. E. Nano letters 2013, 13, 5608-5614.

(14) Trappmann, B.; Gautrot, J. E.; Connelly, J. T.; Strange, D. G.; Li, Y.; Oyen, M. L.; Stuart, M. A. C.; Boehm, H.; Li, B.; Vogel, V.; Spatz, J. Nature materials 2012, 11, 642-649.

(15) Hampe, N.; Jonas, T.; Wolters, B.; Hersch, N.; Hoffmann, B.; Merkel, R. Soft matter 2014, 10, 2431-2443.

(16) MacNearney, D.; Mak, B.; Ongo, G.; Kennedy, T. E. Juncker, D. Langmuir 2016, 32, 13525-13533.

(17) Platzman, I.; Gadomska, K. M.; Janiesch, J.-W.; Louban, I.; Cavalcanti-Adam, E. A.; Spatz, J. P. Methods in cell biology 2013, 119, 237-260

(18) Graeter, S. V.; Huang, J.; Perschmann, N.; López-García, M.; Kessler, H.; Ding, J.; Spatz, J. P. Nano letters 2007, 7, 14131418.

(19) Aydin, D.; Louban, I.; Perschmann, N.; BluİĹmmel, J.; LohmuIILller, T.; Cavalcanti-Adam, E. A.; Haas, T. L.; Walczak, H.; Kessler, H.; Fiammengo, R. Langmuir 2010, 26, $15472-15480$

(20) Carpi, N.; Piel, M.; Azioun, A.; Cuvelier, D.; Fink, J. Protoc. Exch 2011, 10.

(21) Vignaud, T.; Ennomani, H.; Théry, M. Methods in cell biology 2013, 120, 93-116.

(22) Kristensen, S. H.; Pedersen, G. A.; Ogaki, R.; Bochenkov, V.; Nejsum, L. N.; Sutherland, D. S. Acta biomaterialia 2013, 9 , $6158-6168$.

(23) Bernard, A.; Renault, J. P.; Michel, B.; Bosshard, H. R.; Delamarche, E. Advanced Materials 2000, 12, 1067-1070.

(24) Bucknall, D. Nanolithography and patterning techniques in microelectronics; Elsevier, 2005.

(25) Li, J.-R.; Shi, L.; Deng, Z.; Lo, S. H.; Liu, G.-y. Biochemistry 2012, 51, 5876-5893.

(26) Lee, K.-B.; Park, S.-J.; Mirkin, C. A.; Smith, J. C.; Mrksich, M. Science 2002, 295, 1702-1705.

(27) Guo, L. J. Journal of Physics D: Applied Physics 2004, 37, R123.

(28) Kumar, A.; Whitesides, G. M. Applied Physics Letters 1993, $63,2002-2004$.

(29) Mrksich, M.; Whitesides, G. M. Trends in biotechnology 1995, $13,228-235$

(30) Whitesides, G. M.; Ostuni, E.; Takayama, S.; Jiang, X.; Ingber, D. E. Annual review of biomedical engineering 2001, 3 , $335-373$.

(31) Schwab, E. H.; Pohl, T. L.; Haraszti, T.; Schwaerzer, G. K.; Hiepen, C.; Spatz, J. P.; Knaus, P.; Cavalcanti-Adam, E. A Nano letters 2015, 15, 1526-1534.

(32) Gautrot, J. E.; MalmstroÎ́Lm, J.; Sundh, M.; Margadant, C.; Sonnenberg, A.; Sutherland, D. S. Nano letters 2014, 14, 39453952.

(33) Geiger, B.; Spatz, J. P.; Bershadsky, A. D. Nature reviews Molecular cell biology 2009, 10, 21-33.

(34) Théry, M. J Cell Sci 2010, 123, 4201-4213.

(35) Cerf, A.; Dollat, X.; Chalmeau, J.; Coutable, A.; Vieu, C. Journal of Materials Research 2011, 26, 336-346.

(36) Du Roure, O.; Saez, A.; Buguin, A.; Austin, R. H.; Chavrier, P.; Siberzan, P.; Ladoux, B. Proceedings of the National Academy of Sciences of the United States of America 2005, 102, 23902395 .

(37) Tien, J.; Chen, C. S. Ieee Eng Med Biol Mag 2002

(38) Pi, F.; Dillard, P.; Limozin, L.; Charrier, A.; Sengupta, K. Nano letters 2013, 13, 3372-3378.

(39) Pi, F.; Dillard, P.; Alameddine, R.; Benard, E.; Wahl, A.: Ozerov, I.; Charrier, A.; Limozin, L.; Sengupta, K. Nano letters 2015, 15, 5178-5184

(40) Ricoult, S. G.; Sanati Nezhad, A.; Knapp-Mohammady, M.; Kennedy, T. E.; Juncker, D. Langmuir 2014, 30, 12002-12010.

(41) Wang, M. S.; Palmer, L. B.; Schwartz, J. D.; Razatos, A. Langmuir 2004, 20,7753-7759.

(42) Butt, H.-J.; Cappella, B.; Kappl, M. Surface science reports 2005, 59, 1-152.

(43) Kazoe, Y.; Sato, Y. Analytical chemistry 2007, 79, 6727-6733.

(44) Kuddannaya, S.; Chuah, Y. J.; Lee, M. H. A.; Menon, N. V.; Kang, Y.; Zhang, Y. ACS applied materials \& interfaces 2013 5, 9777-9784.

(45) Dillard, P.; Varma, R.; Sengupta, K.; Limozin, L. Biophysical journal 2014, 107, 2629-2638. 